\title{
Alpha-PET with terbium-149: evidence and perspectives for radiotheragnostics
}

\author{
Cristina Müller ${ }^{1 *}$, Christiaan Vermeulen ${ }^{1}$, Ulli Köster ${ }^{2}$, Karl Johnston ${ }^{3}$, Andreas Türler ${ }^{4,5}$, Roger Schibli ${ }^{1,6}$ \\ and Nicholas P. van der Meulen ${ }^{1,4^{*}}$
}

\footnotetext{
* Correspondence: cristina.mueller@ psi.ch; nick.vandermeulen@psi.ch ${ }^{1}$ Center for Radiopharmaceutical Sciences ETH-PSI-USZ, Paul Scherrer Institut, Villigen-PSI, Switzerland Full list of author information is available at the end of the article
}

\begin{abstract}
${ }^{149} \mathrm{~Tb}$ represents a powerful alternative to currently used a-emitters: the relatively short half-life $\left(\mathrm{T}_{1 / 2}=4.1 \mathrm{~h}\right.$ ), low a-energy (3.97 MeV, $\left.\mathrm{l}_{\alpha}=16.7 \%\right)$, absence of a-emitting daughters and stable coordination via DOTA are favorable features for potential clinical application. In this letter, we wish to highlight the unique characteristics of ${ }^{149} \mathrm{~Tb}$ for PET imaging, based on its positron emission $\left(\mathrm{E}_{\beta+\text { mean }}=730 \mathrm{keV}, \mathrm{I}_{\beta+}=7.1 \%\right.$ ) in addition to it's a therapeutic value. To this end, a preclinical study with a tumor-bearing mouse is presented. The perspective of alpha-PET makes ${ }^{149} \mathrm{~Tb}$ highly appealing for radiotheragnostic applications in future clinical trials.
\end{abstract}

Keywords: ${ }^{149} \mathrm{~Tb}$, radiolanthanide, mass separation, PET imaging, a-radionuclide therapy, DOTANOC, AR42J tumor

\section{Correspondence/Findings}

Dear Editor

Recently, $\alpha$-radionuclide therapy has gained in popularity and attracted the interest of physicians [1, 2]. One of the reasons is certainly the success of Xofigo ${ }^{\mathrm{TM}}\left({ }^{223} \mathrm{RaCl}_{2}\right)$, which has been approved for the treatment of patients with symptomatic bone lesions in castration-resistant prostate cancer [3]. The survival benefit after Xofigo ${ }^{\mathrm{TM}}$ therapy, combined with its low toxicity, make it undoubtedly very promising as a novel treatment option for this type of disease and, in future, also possibly for patients suffering from bone metastases of other cancer types [3]. Also, the power of targeted therapy with other $\alpha$-particle emitters, namely, ${ }^{211} \mathrm{At},{ }^{225} \mathrm{Ac}$ and ${ }^{213} \mathrm{Bi}$ has been convincingly demonstrated, mostly in combination with tumor-targeted antibodies [4].

$\alpha$-Particles are positively-charged and have a shorter tissue range $(\sim 25-80 \mu \mathrm{m})$ and much higher energies ( 4-8 MeV), as compared to the negatively-charged $\beta^{-}$-particles of clinically useful $\beta^{-}$-emitters [1]. In terms of radiobiological effects, it is important to mention that the linear energy transfer (LET) of $\alpha$-particles is with $\sim 100 \mathrm{keV} / \mu \mathrm{m}$ very high and may further increase up to $300 \mathrm{keV} / \mu \mathrm{m}$ towards the end of the track (Bragg peak) [5]. This value is far above the LET $(\sim 0.2 \mathrm{keV} / \mu \mathrm{m})$ of $\beta$-particles. High-LET radiation generally causes more lethal cell damage than low-LET radiation due to the formation of more irreparable double-strand DNA breaks [6]. All these characteristics make targeted $\alpha$-therapy best suited for specific tumor cell killing, without collateral damage of surrounding healthy tissue $[1,7]$. Single photon emission computed tomography (SPECT) 
may be used to image targeted $\alpha$-particle therapy if the decay of the $\alpha$-emitting radionuclide results in photon emission of a suitable energy and sufficient intensity [4]. This is the case for ${ }^{223} \mathrm{Ra}\left(\mathrm{E}_{\gamma}=144 \mathrm{keV}, 3.3 \% ; 154 \mathrm{keV}, 5.7 \%\right)$ [8], while ${ }^{225}$ Ac may potentially be imaged via $\gamma$-ray emission of daughter radionuclides (e.g. ${ }^{221} \mathrm{Fr}, \mathrm{E}_{\gamma}=218 \mathrm{keV}, 11 \%$ ), as shown in preclinical settings [9], and ${ }^{211}$ At through use of emitted X-rays [10]. The low quantities of activity employed for $\alpha$-therapy using these radionuclides, remain, however, challenging for nuclear imaging using SPECT.

${ }^{149} \mathrm{~Tb}$ represents a powerful alternative to the currently-employed $\alpha$-emitters. It decays with a relatively short half-life of $4.1 \mathrm{~h}$ and emits $\alpha$-particles of low energy $\left(E_{\alpha}=3.97 \mathrm{MeV}, \mathrm{I}_{\alpha}=16.7 \%\right.$ ), resulting in a tissue range of $\sim 25 \mu \mathrm{m}$ and a LET of $140 \mathrm{keV} / \mu \mathrm{m}$ [11]. These physical properties make it particularly well suited for application in combination with small-molecular-weight targeting agents, including peptides, which are quickly cleared from the body [12]. The absence of $\alpha$-emitting daughters is regarded as an additional favorable feature of ${ }^{149} \mathrm{~Tb}$, since toxicity of $\alpha$-emitters with multiple $\alpha$-emitting daughters has been identified as an issue for clinical application [2]. In vivo application of ${ }^{149} \mathrm{~Tb}$ may, thus, be feasible without the risk of unspecific emission of harmful $\alpha$-particles in the body as a consequence of released daughter radionuclides. The decay scheme of ${ }^{149} \mathrm{~Tb}$ is, nevertheless, complex [7] and potential radiotoxicity of the resulting radiolanthanides remains to be determined. As a radiolanthanide ${ }^{149} \mathrm{~Tb}$ can be stably coordinated with the conventional macrocyclic 1,4,7,10-tetraazacyclododecane-1,4,7,10-tetraacetic acid (DOTA) chelator $[13,14]$. These circumstances allow the use of ${ }^{149} \mathrm{~Tb}$ with DOTA-functionalized compounds that are (pre)clinically established for ${ }^{177} \mathrm{Lu}$-based radionuclide therapy.

Beyer et al. reported on a preclinical immunotherapy with ${ }^{149} \mathrm{~Tb}$-labeled rituximab (5.5 MBq/mouse) in a mouse model of Daudi cell-based lymphoma [15]. In this case, the open-chained cyclohexane diethylene triamine pentaacetic acid (CHX-A"DTPA) chelator was used for radiometal coordination. The majority (89 \%) of treated mice showed tumor-free survival over $>120$ days, while all untreated controls and mice which received unlabeled rituximab developed lymphoma disease [15]. Our group has previously reported on a preclinical pilot study using a ${ }^{149} \mathrm{~Tb}-$ labeled folate conjugate for therapy of mice bearing folate receptor-positive $\mathrm{KB}$ tumor xenografts [14]. In treated mice (2.2 MBq and 3.0 MBq/mouse, respectively), the tumor growth was significantly delayed which prolonged the average survival time to 30.5 days and 43 days, respectively, compared to untreated controls which survived only 21 days on average [14].

Even though the number of preclinical studies in which ${ }^{149} \mathrm{~Tb}$ was investigated, is very small, there is clear evidence of the potential to use ${ }^{149} \mathrm{~Tb}$ for targeted $\alpha$ radionuclide therapy. Other than the aforementioned favorable physical and chemical characteristics it provides, it would also offer a unique opportunity for comparing the effects of $\alpha$-radionuclide therapy with $\beta^{-}$-radionuclide therapy through the use of chemically identical radiopharmaceuticals labeled with ${ }^{149} \mathrm{~Tb}$ and ${ }^{161} \mathrm{~Tb}\left(\mathrm{~T}_{1 / 2}=6.9 \mathrm{~d}\right.$, $\mathrm{E}_{\beta \text {-mean }}=154 \mathrm{keV}$ ) [13]. As all of these features of ${ }^{149} \mathrm{~Tb}$ are so attractive from a therapy perspective, the proposed possibility of positron emission tomography (PET) provides an extra dimension. Alternatively, ${ }^{149} \mathrm{~Tb}$ may also be used for SPECT imaging based on the emission of $\gamma$-radiation of a suitable energy and 
reasonable intensity $\left(E_{\gamma}=165 \mathrm{keV}, \mathrm{I}_{\gamma}=26.4 \%\right)$. This concept has been proposed earlier [16], but has to date not been investigated in preclinical studies. The current trend in nuclear medicine is, however, in favor of PET instead of SPECT due to the higher sensitivity and resolution it provides [17].

In a recently-performed study, we focused on the potentially unique characteristic of ${ }^{149} \mathrm{~Tb}$ to be used for PET imaging, based on its positron emission $\left(\mathrm{E}_{\beta+\text { mean }}=730 \mathrm{keV}\right.$, $\mathrm{I}_{\beta+}=7.1 \%$ ), in addition to its $\alpha$-therapeutic value [16]. ${ }^{149} \mathrm{~Tb}$ was produced by protoninduced spallation of a tantalum target, followed by an online isotope separation process at ISOLDE/CERN (Geneva, Switzerland). The mass-separated ion beam was implanted into a zinc-coated gold catcher foil, which was shipped to Paul Scherrer Institut (PSI, Villigen-PSI, Switzerland) for processing. ${ }^{149} \mathrm{~Tb}$ was separated from isobar and pseudo-isobar impurities by cation exchange chromatography, as previously reported [13]. The separation yield was $100 \mathrm{MBq}(\sim 99 \%)$ of highly pure ${ }^{149} \mathrm{~Tb}$ in $\alpha-$ hydroxyisobutyric acid solution ( $\mathrm{pH} 4.7)$, a quantity sufficient for preclinical application. The radiolabeling was carried out directly in the eluent solution by addition of DOTANOC and incubation of the reaction mixture for $15 \mathrm{~min}$ at $95{ }^{\circ} \mathrm{C} .{ }^{149} \mathrm{~Tb}$-DOTANOC was obtained with $>98 \%$ radiochemical purity at a high specific activity ( $5 \mathrm{MBq} / \mathrm{nmol})$, as confirmed by high performance liquid chromatography (HPLC)-based quality control. A nude mouse bearing AR42J tumor xenografts was intravenously injected with $\sim 7 \mathrm{MBq}{ }^{149} \mathrm{~Tb}-\mathrm{DOTANOC}(\sim 1.4 \mathrm{nmol})$. PET/CT scans were performed $2 \mathrm{~h}$ later using a preclinical G8 bench-top scanner (Sofie Biosciences). During the PET scan $(30 \mathrm{~min})$ and the following CT $(1.5 \mathrm{~min})$ the mouse was anesthesized using a mixture of isoflurane and oxygen.

The quality of the obtained PET images was unexpectedly high (Fig. 1). The maximal intensity projections allowed distinct visualization of the tumors located on each shoulder (Fig. 1a/b). Specific cross sections of the tumor showed homogenous distribution of radioactivity accumulation (Fig. 1c). Residual radioactivity was found in the kidneys and the urinary bladder as expected, based on the fast renal excretion of DOTANOC.

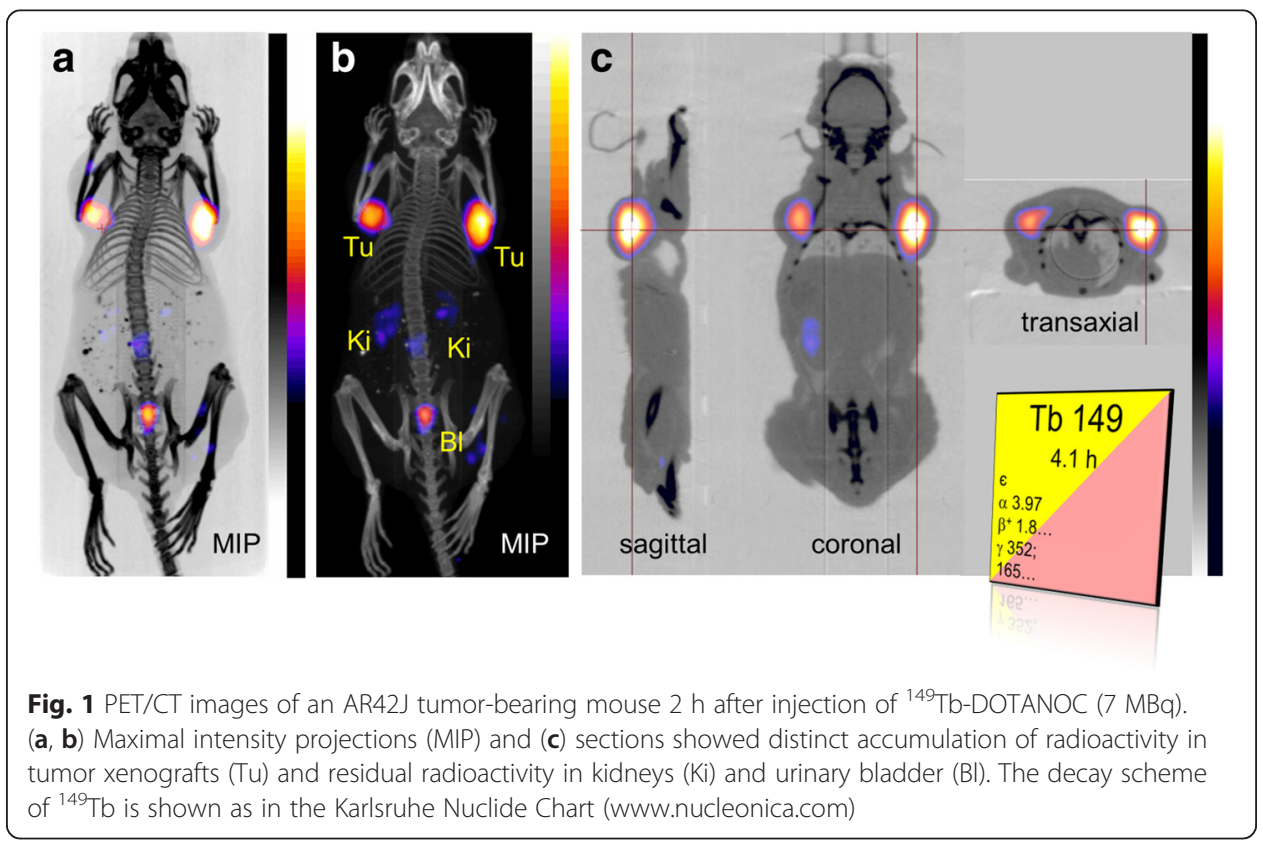


In this study, the possibility of being able to produce a PET image using a ${ }^{149} \mathrm{~Tb}$ labeled biomolecule was successfully demonstrated. It is, thus, indisputable that ${ }^{149} \mathrm{~Tb}$ presents an exceptional potential to be used in clinics as it would allow combining $\alpha$ therapy with PET using a single radionuclide.

It has to be acknowledged, however, that the quantity of injected activity for patients may be critical for PET imaging purposes. So far, it is unknown how much activity would be required for a therapeutic application in the clinics. Among several parameters, including the sensitivity of tumor type which should be treated, it will be critically dependent on the targeting agent and the degree of its accumulation in the tumor tissue. Whether the necessary quantity of radioactivity would allow for PET imaging remains to be determined in patients.

The unconventional production of ${ }^{149} \mathrm{~Tb}$ appeared to be the main reason why ${ }^{149} \mathrm{~Tb}$ did not yet reach clinical trials, as stated in several reports previously [12]. Currently, endeavors all over the world are focused on the establishment of new radionuclide production centers, clearly offering new perspectives for the production of radionuclides like ${ }^{149} \mathrm{~Tb}$, which are dependent on mass separation facilities. Such production centers, which exploit spallation production combined with isotope separation on-line (ISOL), are already in operation at the Isotope Separator and Accelerator (ISAC) at TRIUMF, Canada's National Laboratory for Particle and Nuclear Physics (Vancouver, Canada) and at Investigation of Radioactive Isotopes on Synchrocyclotron (IRIS), at the Petersburg Nuclear Physics Institute (PNPI, Gatchina, Russia). Other facilities are in the planning stage or under construction at Radioactive Isotope Beam Factory (RIBF, East Lansing, U.S.), at the Belgium Nuclear Research Center's ISOL facility (ISOL@MYRRHA, Mol, Belgium) and at the Japan Proton Accelerator Research Complex (J-PARC ISOL, Tokai, Japan). MEDICIS, a new radionuclide production center dedicated to medical applications, is currently being built at CERN (Geneva, Switzerland) [12]. MEDICIS' aim is to produce medically interesting, but not yet fully investigated radionuclides, including ${ }^{149} \mathrm{~Tb}$, in quantities sufficient to address the requirements of pilot investigations in patients.

The perspective of overcoming the obstacle of production holds great promise for more detailed preclinical investigations and first clinical trials in the near future using ${ }^{149} \mathrm{~Tb}$ for $\alpha$-therapy, combined with PET.

\title{
Competing interests
}

None of the authors listed above declares to have any conflict of interest.

Authors' contributions

CM designed and performed the PET animal studies and drafted the manuscript. EV, UK and KJ performed the mass separation at the ISOLDE facility and reviewed the manuscript. AT and RS reviewed the manuscript. NvdM separated the

${ }^{149} \mathrm{~Tb}$ from the target material at PSI and revised the manuscript. All authors read and approved the final manuscript.

\begin{abstract}
Acknowledgments
The authors would like to thank staff members at PSI for technical assistance of the animal experiments, the RILIS team for excellent Dy ionization and the ISOLTRAP-MRTOF-MS team for the on-line measurement of the beam composition as essential information to optimize the collection conditions.
\end{abstract}

Compliance with Ethical Standards

Ethical approval

All applicable international, national, and/or institutional guidelines for the care and use of animals were followed.

This article does not contain any studies with human participants performed by any of the authors.

Author details

${ }^{1}$ Center for Radiopharmaceutical Sciences ETH-PSI-USZ, Paul Scherrer Institut, Villigen-PSI, Switzerland. ${ }^{2}$ Institut

Laue-Langevin, Grenoble, France. ${ }^{3}$ Physics Department, ISOLDE/CERN, Geneva, Switzerland. ${ }^{4}$ Laboratory of 
Radiochemistry, Paul Scherrer Institut, Villigen-PSI, Switzerland. ${ }^{5}$ Department of Chemistry and Biochemistry, University of Bern, Bern, Switzerland. ${ }^{6}$ Department of Chemistry and Applied Biosciences, ETH Zurich, Zurich, Switzerland.

Received: 8 January 2016 Accepted: 11 March 2016

Published online: 28 March 2016

\section{References}

1. Mulford DA, Scheinberg DA, Jurcic JG. The promise of targeted a-particle therapy. J Nucl Med. 2005;46 Suppl 1: 199S-204S.

2. de Kruijff RM, Wolterbeek HT, Denkova AG. A critical review of alpha radionuclide therapy-how to deal with recoiling daughters? Pharmaceuticals (Basel). 2015;8(2):321-36. doi:10.3390/ph8020321.

3. Nilsson S. Alpha-emitter radium-223 in the management of solid tumors: current status and future directions. Am Soc Clin Oncol Educ Book. 2014:e132-9. doi:10.14694/EdBook_AM.2014.34.e132.

4. Wadas TJ, Pandya DN, Solingapuram Sai KK, Mintz A. Molecular targeted alpha-particle therapy for oncologic applications. AJR Am J Roentgenol. 2014;203(2):253-60. doi:10.2214/AJR.14.12554.

5. Kassis Al. Therapeutic radionuclides: biophysical and radiobiologic principles. Semin Nucl Med. 2008;38(5):358-66. doi:10.1053/j.semnuclmed.2008.05.002.

6. Kampf G. Induction of DNA double-strand breaks by ionizing radiation of different quality and their relevance for cell inactivation. Radiobiol Radiother (Berl). 1988;29(6):631-58.

7. Brechbiel MW. Targeted a-therapy: past, present, future? Dalton Trans. 2007;43:4918-28. doi:10.1039/b704726f

8. Hindorf C, Chittenden S, Aksnes AK, Parker C, Flux GD. Quantitative imaging of ${ }^{223}$ Ra-chloride (Alpharadin) for targeted alpha-emitting radionuclide therapy of bone metastases. Nucl Med Commun. 2012;33(7):726-32. doi:10. 1097/MNM.0b013e328353bb6e.

9. McLaughlin MF, Woodward J, Boll RA, Wall JS, Rondinone AJ, Kennel SJ, et al. Gold coated lanthanide phosphate nanoparticles for targeted alpha generator radiotherapy. PLoS One. 2013;8(1):e54531. doi:10.1371/ journal.pone.0054531.

10. Turkington TG, Zalutsky MR, Jaszczak RJ, Garg PK, Vaidyanathan G, Coleman RE. Measuring astatine-211 distributions with SPECT. Phys Med Biol. 1993;38(8):1121-30.

11. Imam SK. Advancements in cancer therapy with alpha-emitters: a review. Int J Radiat Oncol Biol Phys. 2001; 51(1):271-8.

12. Guerard F, Barbet J, Chatal JF, Kraeber-Bodere F, Cherel M, Haddad F. Which radionuclide, carrier molecule and clinical indication for alpha-immunotherapy? Q J Nucl Med Mol Im. 2015;59(2):161-7.

13. Müller C, Zhernosekov K, Köster U, Johnston K, Dorrer H, Hohn A, et al. A unique matched quadruplet of terbium radioisotopes for PET and SPECT and for $\alpha$ - and $\beta$-radionuclide therapy: an in vivo proof-of-concept study with a new receptor-targeted folate derivative. J Nucl Med. 2012;53(12):1951-9. doi:10.2967/jnumed.112.107540.

14. Müller C, Reber J, Haller S, Dorrer H, Köster U, Johnston K, et al. Folate receptor targeted alpha-therapy using terbium-149. Pharmaceuticals (Basel). 2014;7(3):353-65. doi:10.3390/ph7030353.

15. Beyer GJ, Miederer M, Vranjes-Duric S, Comor JJ, Kunzi G, Hartley O, et al. Targeted alpha therapy in vivo: direct evidence for single cancer cell kill using ${ }^{149} \mathrm{~Tb}$-rituximab. Eur J Nucl Med Mol Imaging. 2004;31(4):547-54. doi:10. 1007/s00259-003-1413-9.

16. Allen BJ. Targeted alpha therapy: evidence for potential efficacy of alpha-immunoconjugates in the management of micrometastatic cancer. Australas Radiol. 1999:43(4):480-6.

17. Rahmim A, Zaidi H. PET versus SPECT: strengths, limitations and challenges. Nucl Med Commun. 2008;29(3):193-207. doi:10.1097/MNM.0b013e3282f3a515.

\section{Submit your manuscript to a SpringerOpen ${ }^{\circ}$ journal and benefit from:}

- Convenient online submission

- Rigorous peer review

- Immediate publication on acceptance

- Open access: articles freely available online

- High visibility within the field

- Retaining the copyright to your article 